\title{
High glucose-induced Matrilin-2 expression in mouse mesangial cells was mediated by transforming growth factor beta 1 (TGF- $\beta 1$ )
}

Shukun Zhang ${ }^{a, 1}$, Menglan Zhang ${ }^{a, 1}$, Hong Huang ${ }^{b}$, Shiying Zhou ${ }^{a}$, Yanshneg Du ${ }^{c}$, Xin $\mathrm{Yi}^{\mathrm{c}, *,}$, Junming Luo ${ }^{\mathrm{a}, *}$

aDepartment of Pathology, Qinghai Provincial People's Hospital, 2 Gonghe Road, Xining, Qinghai 810007, China

${ }^{\mathrm{b}}$ Medical College of Qinghai University, 16 Kunlun Road, Xining 810000, China 'Department of Neurology, Indiana University School of Medicine, Indianapolis, IN 46202, USA

${ }^{1}$ Two authors contribute equally to this work.

*Correspondence should be addressed to Xin Yi; xinyi@iupui.edu and Junming Luo; jluo099@163.com

This is the author's manuscript of the article published in final edited form as:

Zhang, S., Zhang, M., Huang, H., Zhou, S., Du, Y., Yi, X., \& Luo, J. (2016). High glucose-induced Matrilin-2 expression in mouse mesangial cells was mediated by transforming growth factor beta 1 (TGF- $\beta 1$ ). Biochemical and Biophysical Research Communications, 474(2), 303-308. https://doi.org/10.1016/j.bbrc.2016.04.091 


\section{Abstract}

This study aimed at evaluating the effect of high glucose on the expression of extracellular matrix (ECM) protein Matrilin-2 and the mechanism underlying this effect by using a mouse mesangial cell line. Mouse mesangial cells (MMCs) were cultured in media containing normal (5mM D-glucose) or high concentrations of glucose (30mM D-glucose). The expression of Matrilin-2 was assessed by either RT-PCR or western blot. Additionally, transforming growth factor beta 1 (TGF- $\beta 1$ ) inhibitors and TGF- $\beta 1$ were used to determine whether glucose-regulated Matrilin- 2 expression was mediated by the TGF- $\beta 1 / \mathrm{Smad} 3$ signaling pathway. Our data demonstrated that Matrilin-2 expression was markedly induced by high glucose and TGF- $\beta 1$. High glucose-induced Matrilin-2 expression was inhibited by TGF- $\beta 1 / S m a d 3$ inhibitors, indicating that Matrilin-2 was markedly induced by high glucose and this induction was mediated by the TGF- $\beta 1 /$ Smad3 pathway. Taken together, our results showed that high-glucose-induced Matrilin-2 expression that was mediated by the TGF- $\beta 1 / \mathrm{Smad} 3$ signaling pathway might play a role in Diabetic nephropathy (DN) pathogenesis and our finding provided a potential diagnostic and/or therapeutic target for DN.

Keywords: Diabetic nephropathy; extracellular matrix; Matrilin-2; Transforming growth factor beta 1 


\section{Introduction}

Diabetic nephropathy (DN) is the leading cause of chronic kidney disease and one of the most significant long-term complications in terms of morbidity and mortality for individual patients with diabetes. Major typical morphological changes are the progressive accumulation of extracellular matrix (ECM) proteins in mesangial interstitial space to lead to glomerular and tubular basement membrane thickening and increase of mesangial matrix, ultimately resulting in glomerulosclerosis and tubulointerstitial fibrosis. ECM is a potential protein source of diagnostic biomarkers for renal fibrosis [1, 2], and among a large number of ECM proteins, Matrilin is a novel filamentous-forming adapter protein family, which can form collagen-dependent and -independent network of extracellular matrix [3-5]. Matrilin-2 is the largest member of the Matrilin family that shares von Willebrand factor A (vWFA) domains, epithelial growth factor (EGF) like repeats, and a series of heptad repeats at their C-terminal coiled-coil domain[6,7]. Unlike other family members whose expression is limited to skeletal tissues, Matrilin-2 is found heterogeneously distributed in connective tissues, including kidney $[8,9]$. It has previously been described that Matrilin-2 can interact with itself and a variety of matrix molecules including type 1 collagen, fibronectin, and laminin and has been postulated to act as an adaptor molecule connecting these molecules with other proteins and proteoglycans within the ECM where it may regulate ECM homeostasis [7, 10]. Therefore, Matrilin-2 may play an important role in DN pathogenesis.

Mesangial cells (MCs) located in the intercapillary space that are activated by 
numerous vasoactive substances as well as growth factors. Among various growth factors contributing to DN pathogenesis, transforming growth factor- $\beta$ (TGF- $\beta$ ) is the key stimulator of ECM accumulation in the kidney [11]. It has been suggested that TGF- $\beta$ significantly contributes to glomerular filtration barrier alteration, fibrosis and sclerosis, which reduce the filtration surface and finally cause glomerular collapse at the tubular level[12]. Studies with mouse models of diabetes showed antibodies against TGF- $\beta$ prevented mesangial matrix accumulation [13]. TGF- $\beta$ exerts its biological effects by binding to complexes of type I and II serine/threonine kinase receptor that further activates downstream mediators, Smad2 and Smad3 [14]. Smad3 is critical for pro-fibrotic effect of TGF- $\beta$ [15-17] and targeting Smad3 may have great therapeutic potential for kidney diseases. Interestingly, recent study showed that Matrilin-2 promoter harbored 7 putative Smad-binding sites [18, 19]. In this study, therefore, we investigated whether hyperglycemia was able to directly stimulate matrilin-2 overexpression and if this process was mediated by the TGF- $\beta /$ Smad 3 pathway by using a mouse mesangial cell line.

\section{Materials and Methods}

\subsection{Cell culture}

Mouse mesangial cells (MMCs) (SV40 MES13) were obtained from the American Type Culture Collection (ATCC, Rockville, MD) and maintained in continuous culture at $37^{\circ} \mathrm{C} / 5 \% \mathrm{CO} 2$ using DMEM/F-12 containing 5\% FBS and $12 \mathrm{mM}$ HEPES $(\mathrm{pH} 7.0)$. MMCs grown in $100 \mathrm{~mm}$ culture dish with similar confluent were incubated with serum-free media for 48 hours to synchronize the cell growth. Then, cells were plated 
into 12-well culture dishes for subsequent assays. To assess the expression of Matrilin-2 cultured under diabetic conditions, MMCs were grown in high glucose (30 $\mathrm{mM}$ ) medium for 24 hours, and harvested for PCR and western blot analysis. For TGF- $\beta 1$ stimulation study, cells were treated with $10 \mathrm{ng} / \mathrm{ml}$ recombinant TGF- $\beta 1$ (PeproTech, Rocky Hill, NJ ), and then were harvested at different time points (12h, $24 \mathrm{~h}, 48 \mathrm{~h}$, and $72 \mathrm{~h}$ ). While for TGF- $\beta 1 / \mathrm{Smad} 3$ signaling pathway assay either in high-glucose or normal condition, cells were pretreated with the indicated amount of the specific TGF receptor type I inhibitor SB-431542 (Selleckchem) and Smad3 inhibitor SIS3 (Billerica MA) for $2 \mathrm{~h}$, followed by stimulation with $10 \mathrm{ng} / \mathrm{ml}$ TGF- $\beta 1$ for $24 \mathrm{~h}$, samples were then measured by Western blotting analysis

\subsection{Real-Time PCR}

Total RNAs were extracted from MMCs using RNeasy Kit (Qiagen, Valencia, CA) according to the manufacturer's instructions. QuantiTect SYBR green PCR Kit (Qiagen, Valencia, CA) with DNA Engine Opicon 2 Continuous Fluorescence Detection System (MJ Research, Waltham, MA) were used to perform quantitative RT-PCR. Primers used in amplification of target genes mRNA were as follows: matrilin-2 (forward, 5'-TGCCTCTGAGCCCATTGACAAG-3'; reverse, 5'-TATGTTGCACTGTTGGCTGGT-3'); $18 S$ RNA (forward, 5'-CGGCTACCACATCCAAGGAA-3'; reverse, 5'-GCTGGAATTACCGCGGCT-3'). The 18S RNA was amplified at the same time and used as an internal control. The matrilin-2 mRNA level was normalized to housekeeping gene 18S RNA levels. Then 
the relative value of matrilin-2 mRNA was measured and calculated.

\subsection{Protein extraction and Western Blotting Analysis}

Western blotting was performed to determine Matrilin-2 protein expression level in MMCs under high-glucose condition. Treated cells were harvested and lysed with RIPA buffer (Sigma, St. Louis, MO). Protein quantification was performed by BCA protein assay (Thermo Scientific, Waltham, MA). Equal amounts of protein (30 ug/lane) were resolved by $12 \%$ SDS-polyacrylamide gels, transferred onto nitrocellulose membranes (Invitrogen, Carlsbad, CA), then the membranes were blocked with 5\% milk in TBST (25 mM Tris-HCl, pH 7.5; $125 \mathrm{mM} \mathrm{NaCl} ; 0.1 \%$ Tween 20), followed by incubation with polyclonal rabbit anti-Matrilin-2 antibody (1:2000, NBP1-76328) (Novus Biologicals, Littleton $\mathrm{CO}$ ) and rabbit anti- $\beta$-actin antibody $(1: 3000,622101)$ (Biolegend, San Diego, CA) at $4^{\circ} \mathrm{C}$ overnight, then the bound horseradish peroxidase-conjugated secondary antibody was detected using an ECL detection system. Protein expression levels were determined by analyzing the signals captured on the nitrocellulose membranes using an image analyzer.

\subsection{Statistics}

Results were presented as the Mean \pm SD. Paired Student's t-tests were used for statistical analysis, $p$ values less than 0.05 were considered significant.

\section{Results}

\subsection{High glucose significantly induced Matrilin-2 expression.}

To investigate whether Matrilin-2 was involved in DN, we observed the mRNA and protein expression levels of Matrilin-2 in SV40 MES 13 cells (MMCs) treated with the 
high concentration of glucose $(30 \mathrm{mM})$. Clearly, both Matrilin-2 mRNA and protein levels were significantly upregulated in a high glucose condition when comparing to normal glucose condition $(p<0.05)$ (Figure $1, A, B, C)$.

3.2 Inhibition of TGF- $\beta 1$ and Smad3 suppressed high-glucose-induced Matrilin-2 expression.

We pre-treated MMCs with the specific TGF type I receptor kinase inhibitor SB-431542, followed by stimulation with $24 \mathrm{~h}$ high glucose treatments. The western blot showed that SB-431542 markedly inhibited high glucose-induced Matrilin-2 expression at 10uM. Additionally, we applied Smad3 specific inhibitor SIS3 to cultured cells and demonstrated that SIS3 significantly inhibited high glucose-induced Matrilin-2 expression at a concentration of $10 \mathrm{uM}$ as well (Figure 2, A, B).

3.2 TGF- $\beta 1$ was able to stimulate expression of Matrilin-2 protein at time-dependent manner in MMCs

For further confirmation, we investigated whether TGF- $\beta 1$ was able to directly induce Matrilin-2 expression in MMCs. Semi-quantitative analysis showed that after cultured MMCs were treated with $10 \mathrm{ng} / \mathrm{ml}$ TGF- $\beta 1$, Matrilin-2 expression was markedly induced in a time-dependent manner (Figure 3.A, B). The expression of Matrilin-2 was significantly elevated starting at $12 \mathrm{~h}$ post TGF- $\beta 1$ treatments $(p<0.05)$ and reached to peak value at $24 \mathrm{~h}(\mathrm{p}<0.01)$.

3.3 TGF- $\beta 1 /$ Smad3 inhibitors inhibited TGF- $\beta 1$-induced Matrilin-2 expression in MMCs.

As expected, both SB-431542 and SIS3 inhibited TGF- $\beta 1$-induced Matrilin-2 
expression (Figure 4. A, B).

\section{Discussion}

In DN, changes in the ECM such as increased glomerular basement membrane thickness and the extracellular matrix of the mesangium expand occur in both type 1 and type 2 diabetes. The mesangial ECM is the main cause of declining renal function in DN provides structural support for the glomerular capillary convolute, connecting with the extraglomerular mesangium at the vascular pole and dysregulation of cell-matrix signaling plays an important role in $\mathrm{DN}[2]$. It was found that hyperglycemia induced TGF-expression in experimental and human diabetes [20, 21]. Previous findings have demonstrated that high glucose stimulates TGF- $\beta 1$ very early through its receptor [22, 23]. It was also demonstrated that TGF-bIIR played an important role in the progression of diabetic nephropathy through the intracellular Smad signaling [24]. Matrilin-2 was proved to be essential adaptor protein for the generation of muscle and nerve by TGF- $\beta /$ Smad signaling pathway [25], although it remained unknown whether Matrilin-2 was involved in DN pathogenesis and if the TGF- $\beta 1$ played a role in regulating this protein. In this study, we clearly demonstrated that Matrilin-2 expression was induced by hyperglycemia and the TGF- $\beta 1 /$ Smad3 pathway was involved in high glucose-induced Matrilin-2 expression in MMCs.

Here, the expression levels of Matrilin-2 mRNA and protein were significantly increased after MMCs had been treated with high glucose, suggesting that Matrilin-2 might be involved in the pathogenesis and progression of DN. Our previous study demonstrated that Matrilin-2 was mainly expressed in glomerulus, the basement 
membrane of distal convoluted tubule and renal matrix [8], where was the typical lesion location in DN. Change of glucose metabolism could therefore activate intraand extracellular signaling pathways leading to abnormal ECM accumulation. This was the first time that Matrilin-2 was proved to be upregulated in MMCs under a high-glucose condition and our data suggested that Matrilin-2 accumulation might be an important damage factor for glomerular fibrosis in DN.

MMCs, locating in the intercapillary space, are vital for glomerulus to maintain normal physiological status. The response of MMCs under hyperglycemia condition is highly complex. Besides being exposed to extracellular glycated-proteins, MMCs activities are also affected and controlled by intracellular mediators, such as TGF- $\beta 1$. In renal diseases, TGF- $\beta 1$ is upregulated and stimulates MMCs to produce ECM proteins, including type I, III and IV collagen, and laminin, contributing to glomerular ECM accumulation $[12,26]$. In MMCs, it has been proved that TGF- $\beta 1 / \mathrm{Smad} 3$ signaling pathway could stimulates the synthesis and accumulation of ECM, and is associated with MMC hypertrophy in diabetes[12]. Here, we demonstrated that TGF- $\beta 1$ and Smad3 mediated high glucose-induced Matrilin-2 activation in MMCs.

The biomarkers of tissue fibrosis have been investigated extensively in the pathogenesis of DN. Here our study revealed that Matrilin-2 could be induced by high glucose in MMCs through the TGF- $\beta 1 /$ Smad3 signaling pathway. Our data may provide a potential diagnostic and therapeutic target for DN.

\section{Conflict of interest}


There is no conflict of interests.

\section{Acknowledgments}

This work was supported by the National Natural Science Foundation of China (NSFC) grant (No. 81560123), and by the Applied Basic Research Plan of Qinghai Province Science and Technology Department (No. 2014-ZJ-731). 


\section{Reference}

[1] M.P. Cohen, G.T. Lautenslager, C.W. Shearman, Increased urinary type IV collagen marks the development of glomerular pathology in diabetic $\mathrm{d} / \mathrm{db}$ mice, Metabolism: clinical and experimental, 50 (2001) 1435-1440.

[2] R.M. Mason, N.A. Wahab, Extracellular matrix metabolism in diabetic nephropathy, J Am Soc Nephrol, 14 (2003) 1358-1373.

[3] A.R. Klatt, A.K. Becker, C.D. Neacsu, M. Paulsson, R. Wagener, The matrilins: modulators of extracellular matrix assembly, Int J Biochem Cell Biol, 43 (2011) 320-330.

[4] S. Dennler, M.J. Goumans, P. ten Dijke, Transforming growth factor beta signal transduction, J Leukoc Biol, 71 (2002) 731-740.

[5] Q. Chen, Y. Zhang, D.M. Johnson, P.F. Goetinck, Assembly of a novel cartilage matrix protein filamentous network: molecular basis of differential requirement of von Willebrand factor A domains, Mol Biol Cell, 10 (1999) 2149-2162.

[6] F. Deak, D. Piecha, C. Bachrati, M. Paulsson, I. Kiss, Primary structure and expression of matrilin-2, the closest relative of cartilage matrix protein within the von Willebrand factor type A-like module superfamily, J Biol Chem, 272 (1997) 9268-9274.

[7] D. Piecha, C. Wiberg, M. Morgelin, D.P. Reinhardt, F. Deak, P. Maurer, M. Paulsson, Matrilin-2 interacts with itself and with other extracellular matrix proteins, Biochem J, 367 (2002) 715-721.

[8] S. Zhang, J. Peng, Y. Guo, S. Javidiparsijani, G. Wang, Y. Wang, H. Liu, J. Liu, J. Luo, Matrilin-2 is a widely distributed extracellular matrix protein and a potential biomarker in the early stage of osteoarthritis in articular cartilage, Biomed Res Int, 2014 (2014) 986127.

[9] S. Frank, T. Schulthess, R. Landwehr, A. Lustig, T. Mini, P. Jeno, J. Engel, R.A. Kammerer, Characterization of the matrilin coiled-coil domains reveals seven novel isoforms, J Biol Chem, 277 (2002) 19071-19079.

[10] A.R. Klatt, D.P. Nitsche, B. Kobbe, M. Macht, M. Paulsson, R. Wagener, Molecular structure, processing, and tissue distribution of matrilin-4, J Biol Chem, 276 (2001) 17267-17275.

[11] T. Yamamoto, T. Nakamura, N.A. Noble, E. Ruoslahti, W.A. Border, Expression of transforming growth factor beta is elevated in human and experimental diabetic nephropathy, Proc Natl Acad Sci U S A, 90 (1993) 1814-1818.

[12] F.J. Lopez-Hernandez, J.M. Lopez-Novoa, Role of TGF-beta in chronic kidney disease: an integration of tubular, glomerular and vascular effects, Cell Tissue Res, 347 (2012) 141-154.

[13] F.N. Ziyadeh, B.B. Hoffman, D.C. Han, M.C. Iglesias-De La Cruz, S.W. Hong, M. Isono, S. Chen, T.A. McGowan, K. Sharma, Long-term prevention of renal insufficiency, excess matrix gene expression, and glomerular mesangial matrix expansion by treatment with monoclonal antitransforming growth factor-beta antibody in db/db diabetic mice, Proc Natl Acad Sci U S A, 97 (2000) 8015-8020.

[14] R. Derynck, Y.E. Zhang, Smad-dependent and Smad-independent pathways in TGF-beta family signalling, Nature, 425 (2003) 577-584.

[15] W. Yuan, J. Varga, Transforming growth factor-beta repression of matrix metalloproteinase-1 in dermal fibroblasts involves Smad3, The Journal of biological chemistry, 276 (2001) 38502-38510. 
[16] E. Piek, W.J. Ju, J. Heyer, D. Escalante-Alcalde, C.L. Stewart, M. Weinstein, C. Deng, R. Kucherlapati, E.P. Bottinger, A.B. Roberts, Functional characterization of transforming growth factor beta signaling in Smad2- and Smad3-deficient fibroblasts, The Journal of biological chemistry, 276 (2001) 19945-19953.

[17] F. Verrecchia, M.L. Chu, A. Mauviel, Identification of novel TGF-beta /Smad gene targets in dermal fibroblasts using a combined cDNA microarray/promoter transactivation approach, The Journal of biological chemistry, 276 (2001) 17058-17062.

[18] F. Deak, L. Mates, E. Korpos, A. Zvara, T. Szenasi, M. Kiricsi, L. Mendler, A. Keller-Pinter, B. Ozsvari, H. Juhasz, L. Sorokin, L. Dux, N. Mermod, L.G. Puskas, I. Kiss, Extracellular deposition of matrilin-2 controls the timing of the myogenic program during muscle regeneration, Journal of cell science, 127 (2014) 3240-3256.

[19] T. Ichikawa, Y. Suenaga, T. Koda, T. Ozaki, A. Nakagawara, DeltaNp63/BMP-7-dependent expression of matrilin-2 is involved in keratinocyte migration in response to wounding, Biochemical and biophysical research communications, 369 (2008) 994-1000.

[20] I.S. Park, H. Kiyomoto, S.L. Abboud, H.E. Abboud, Expression of transforming growth factor-beta and type IV collagen in early streptozotocin-induced diabetes, Diabetes, 46 (1997) 473-480.

[21] C. Weigert, K. Brodbeck, K. Klopfer, H.U. Haring, E.D. Schleicher, Angiotensin II induces human TGF-beta 1 promoter activation: similarity to hyperglycaemia, Diabetologia, 45 (2002) 890-898.

[22] H. Sakai, K. Jinde, D. Suzuki, M. Yagame, Y. Nomoto, Localization of glycated proteins in the glomeruli of patients with diabetic nephropathy, Nephrology, dialysis, transplantation : official publication of the European Dialysis and Transplant Association - European Renal Association, 11 Suppl 5 (1996) 66-71.

[23] C.R. Ban, S.M. Twigg, Fibrosis in diabetes complications: pathogenic mechanisms and circulating and urinary markers, Vascular health and risk management, 4 (2008) 575-596.

[24] J.D. Mott, R.G. Khalifah, H. Nagase, C.F. Shield, 3rd, J.K. Hudson, B.G. Hudson, Nonenzymatic glycation of type IV collagen and matrix metalloproteinase susceptibility, Kidney international, 52 (1997) 1302-1312.

[25] E. Korpos, F. Deak, I. Kiss, Matrilin-2, an extracellular adaptor protein, is needed for the regeneration of muscle, nerve and other tissues, Neural regeneration research, 10 (2015) 866-869.

[26] G. Gruden, P.C. Perin, G. Camussi, Insight on the pathogenesis of diabetic nephropathy from the study of podocyte and mesangial cell biology, Current diabetes reviews, 1 (2005) 27-40. 
Figures
Figure 1
A

Figures
Figure 1
A

A

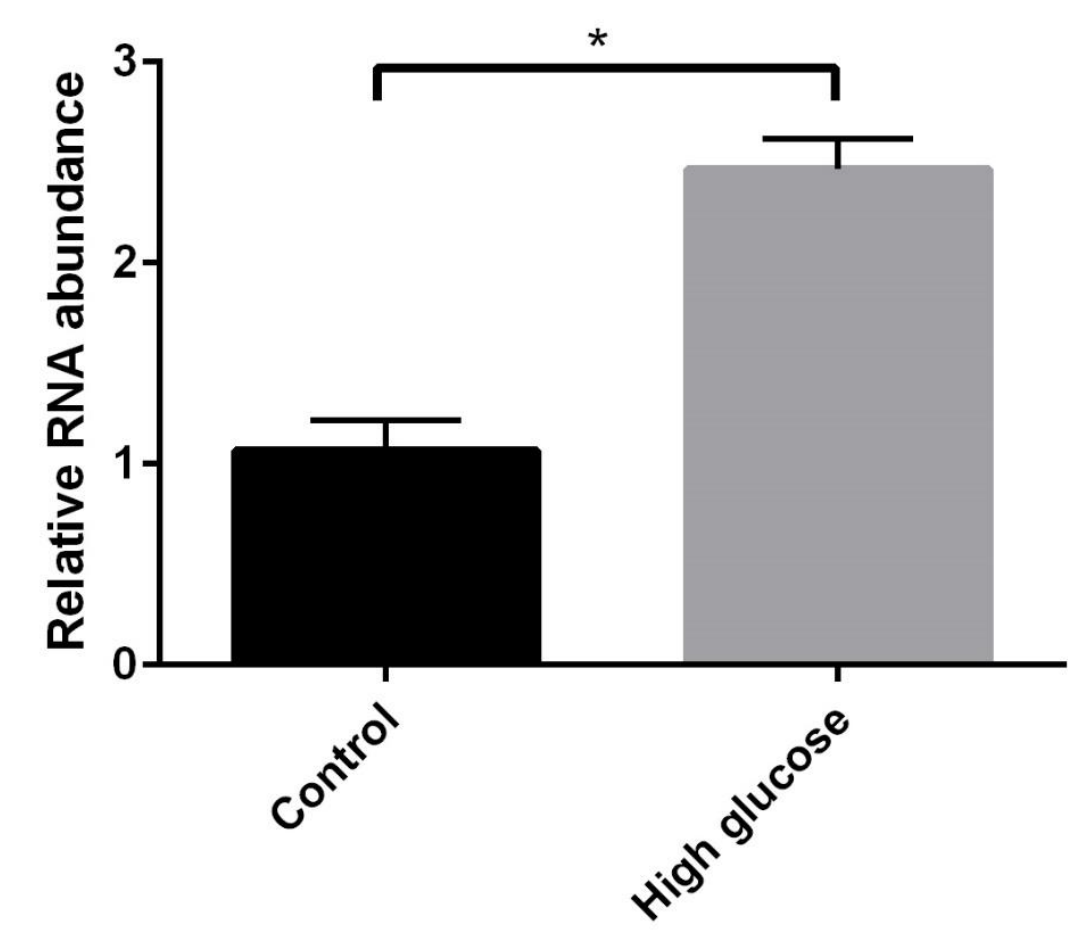


Control

$\longrightarrow$
High glucose

Matrilin-2

$\beta$-actin

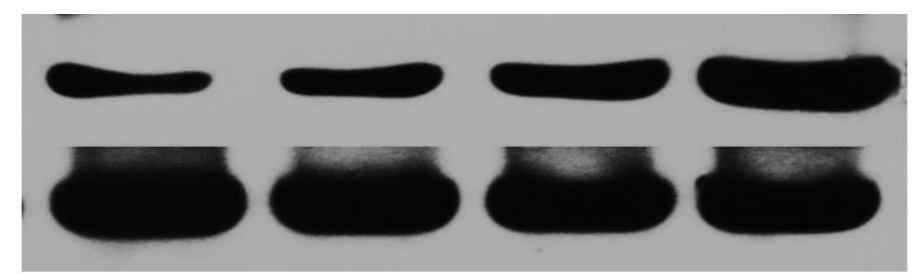

C

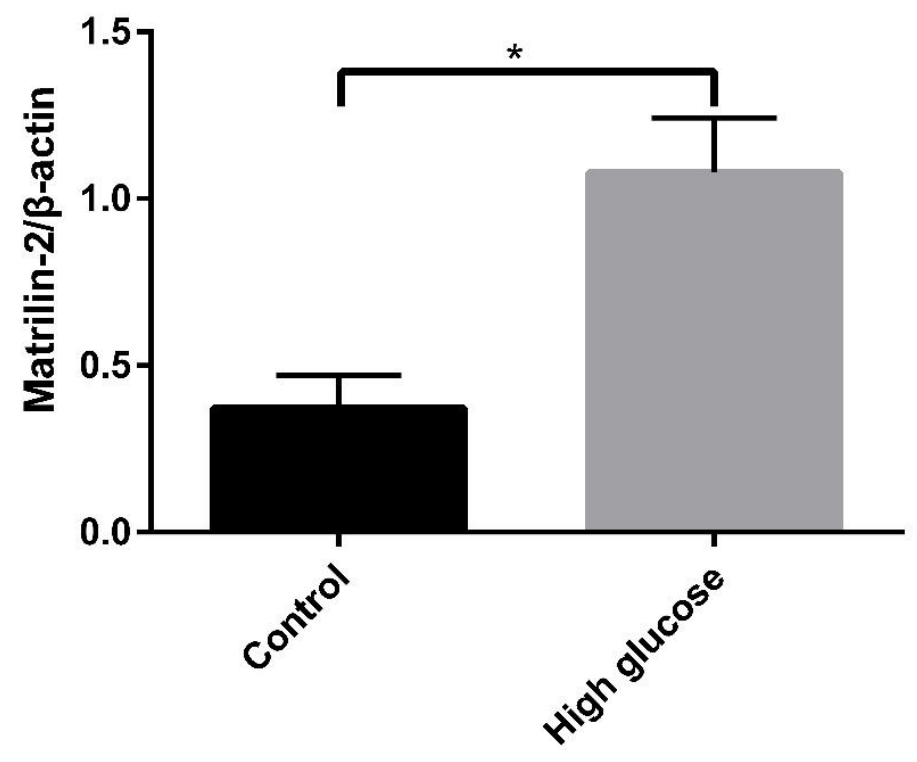


Figure 2

A

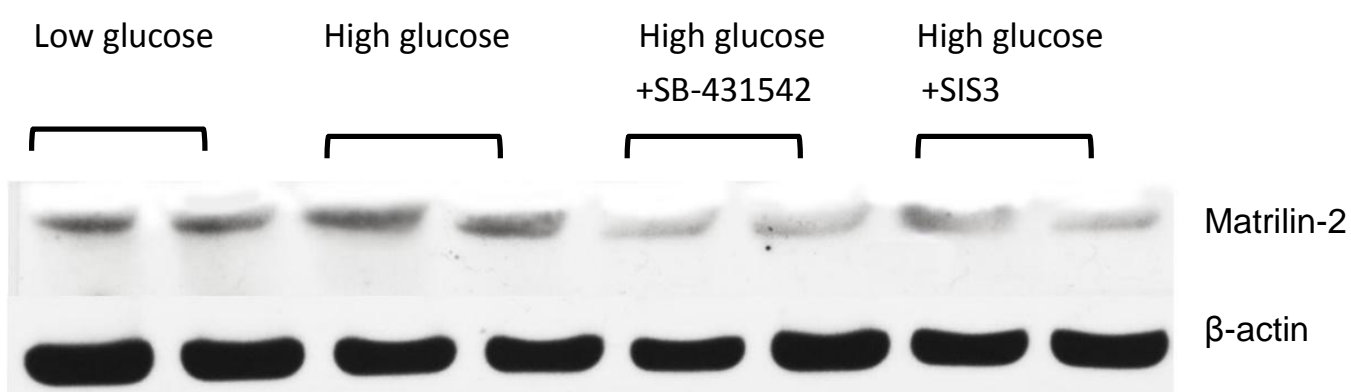

B

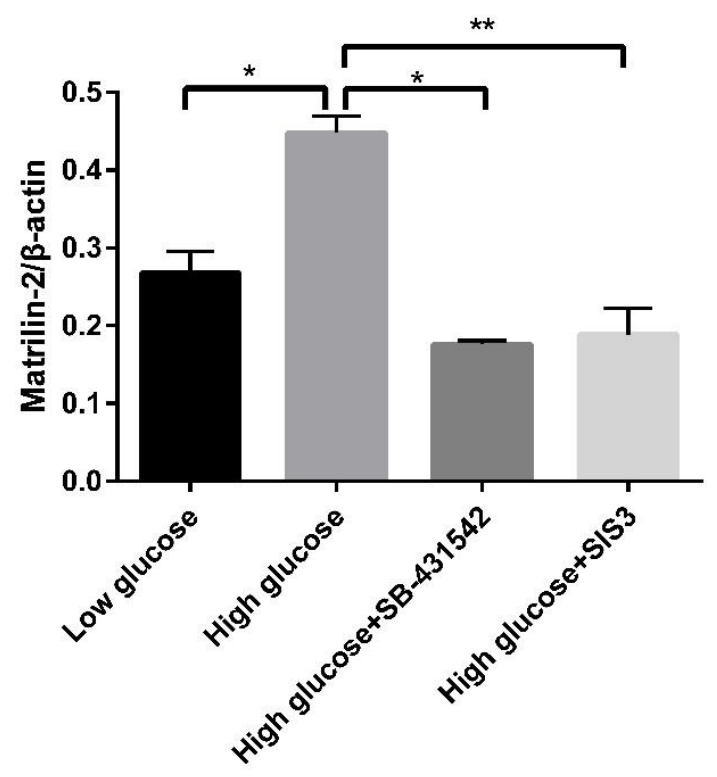


Figure3

A
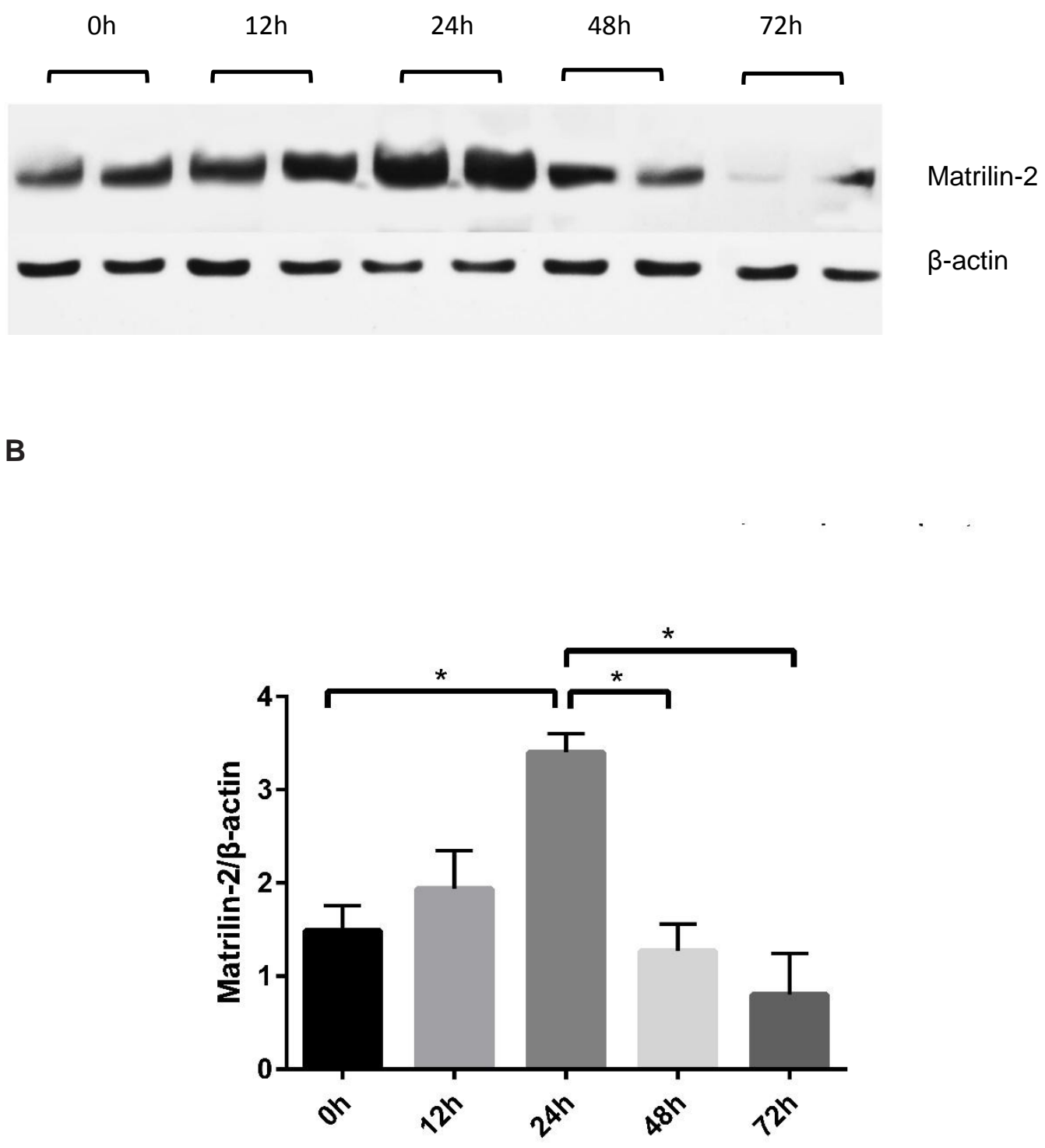
Figure 4

A
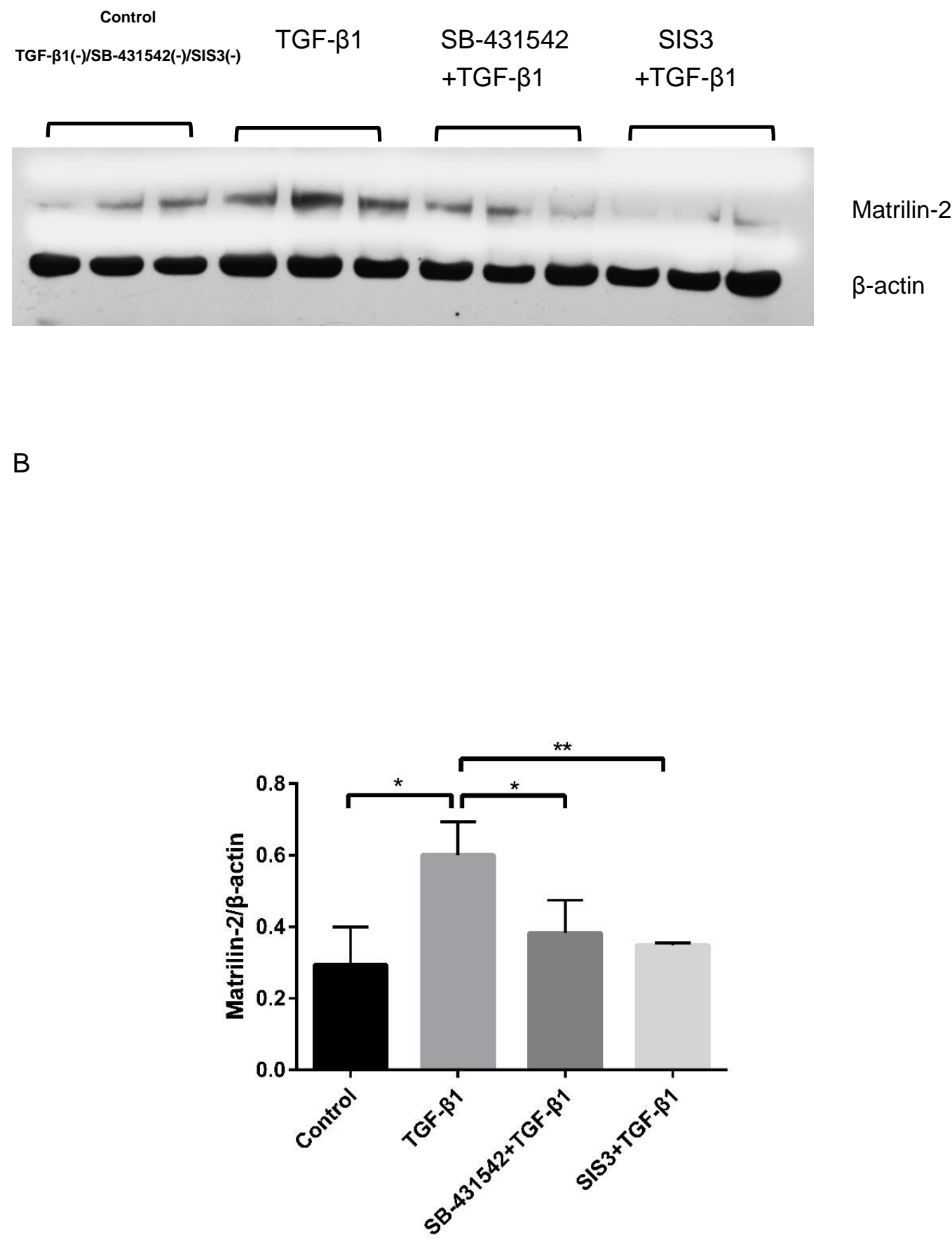


\section{Figure Legends}

Figure 1. High glucose induced mRNA and protein expression of matrilin-2 in MMCs. A, PCR results showed that Matrilin-2 mRNA expression levels were significantly upregulated when MMCs had been treated with high glucose. B. Western blot demonstrated that Matrilin-2 protein bands at $74 \mathrm{kD}$ was markedly increased by high glucose $(30 \mathrm{mM})$ as compared to the control group. C, semi-quantitative result showed that Matrilin-2 protein expression levels were significantly induced by high glucose as comparing to the control group. ${ }^{*} \mathrm{p}<0.05$

\section{Figure 2.}

High glucose-induced Matrilin-2 was suppressed by TGF- $\beta 1$ and Smad3 inhibitors. A, monolayer MMCs grown in complete growth medium were pretreated with specific TGF receptor type I inhibitor, SB-431542 (10uM), and Smad3 inhibitor, SIS3 (10 uM), for $2 \mathrm{~h}$ followed treatments with normal (5mM) or high glucose $(30 \mathrm{mM})$ for additional $24 \mathrm{~h}$. Western blotting analyses were then used for Matrinlin-2 quantification. B, ImageJ software analysis showed that TGF- $\beta 1$ and Smad3 inhibitors significantly suppressed high glucose-induced Matrilin-2 protein expression. ${ }^{*} p<0.05,{ }^{* *} p<0.01$.

\section{Figure 3.}


TGF- $\beta 1$ stimulated Matrilin-2 expression in a time-dependent manner. A, MMCs had been treated with $10 \mathrm{ng} / \mathrm{ml}$ TGF- $\beta 1$ for the indicated time period. The protein levels of Matrilin-2 were measured by using Western blot analyses. B, Quantitation analyses showed Matrilin-2 expression levels were induced starting at $12 \mathrm{~h}$ post TGF- $\beta 1$ treatments and reached to peak value at $24 \mathrm{~h} .{ }^{*} \mathrm{p}<0.05$, ${ }^{* *} \mathrm{p}<0.01$.

Figure 4. TGF- $\beta 1$ and Smad3 inhibitors suppressed TGF- $\beta 1$-induced Matrilin-2 expression. A. MMCs were pretreated with specific TGF receptor type I inhibitor, SB-431542 (10uM), and Smad3 inhibitor, SIS3 (10uM), for 2h, followed by treatments with TGF- $\beta 1(10 \mathrm{ng} / \mathrm{mL})$ for additional $24 \mathrm{~h}$. The expression levels of Matrilin-2 were determined by Western blot analyses. B, Semi-quantitative analyses by ImageJ showed that TGF- $\beta 1$ significantly stimulated Matrilin-2 protein expression as compared with the control group $(p<0.05)$. MMCs pretreated with TGF- $\beta 1$ and Smad3 inhibitors inhibited TGF- $\beta 1$-induced Matrilin-2 expression $\left({ }^{*} p<0.05,{ }^{* *} p<0.01\right)$. 